\title{
The Eating Disorder Inventory in the screening for DSM-5 binge eating disorder
}

\author{
Linda Mustelin a,b,c,*, Ulla Kärkkäinen a , Jaakko Kaprio a,c, Anna Keski-Rahkonen a \\ a Department of Public Health, University of Helsinki, Finland \\ ${ }^{\mathrm{b}}$ Department of Psychiatry, University of North Carolina at Chapel Hill, Chapel Hill, NC, United States \\ c Institute for Molecular Medicine Finland FIMM, University of Helsinki, Helsinki, Finland
}

\section{A R T I C L E I N F O}

\section{Article history:}

Received 13 November 2015

Received in revised form 13 April 2016

Accepted 1 June 2016

Available online 3 June 2016

\section{Keywords:}

Binge eating disorder

Screening

Sensitivity and specificity

Eating Disorder Inventory

Community-based study

\begin{abstract}
A B S T R A C T
Background: We assessed whether the Eating Disorder Inventory (EDI) is suitable for screening binge eating disorder (BED) in young women.

Method: Young women ( $\mathrm{N}=2825)$ from the 1975-79 birth cohorts of Finnish twins were assessed by questionnaires, including subscales of the EDI. For a subset of women ( $N=548$ ), we established DSM-5 diagnoses of BED; 16 women had lifetime BED. We compared screening properties of the EDI scales using receiver operating characteristic (ROC) analysis, determined optimal cutoff points, and calculated sensitivities and specificities.

Results: The best screen for DSM-5 BED was the global score of three subscales (Bulimia, Drive for Thinness, Body Dissatisfaction) with an area under the curve (AUC) of 0.86 . Its sensitivity was $87 \%$ and specificity $76 \%$ at cutoff $\geq 21$. Three individual subscales had acceptable screening properties: Bulimia (AUC 0.83 ; sensitivity $80 \%$, specificity $78 \%$ at cutoff $\geq 2$ ), Drive For Thinness (AUC 0.82 ; sensitivity $87 \%$, specificity $72 \%$ at cutoff $\geq 7$ ), and Body Dissatisfaction (AUC 0.81 ; sensitivity $93 \%$, specificity $60 \%$ at cutoff $\geq 8$ ).

Conclusion: The EDI performed well as a screening tool for BED in our community-based sample of young twin women. Future studies should assess its value in other populations and in clinical settings.
\end{abstract}

(c) 2016 Elsevier Ltd. All rights reserved.

\section{Introduction}

Binge eating disorder (BED) is characterized by episodic binge eating in the absence of regular compensatory behaviors such as vomiting (American Psychiatric Association, 2013). It is associated with high levels of obesity (Bulik, Sullivan, \& Kendler, 2002; Striegel-Moore et al., 2001) and psychiatric comorbidity, particularly major depression (Bulik et al., 2002; Mustelin, Raevuori, Hoek, Kaprio, \& Keski-Rahkonen, 2015). Although effective treatments are available (Berkman et al., 2015), BED often goes undetected and untreated (Mond, Hay, Rodgers, \& Owen, 2007; Mustelin et al., 2015). Because BED can have serious long-term health consequences, such as obesity and related metabolic disturbances (Hudson et al., 2010; Mussell et al., 1995) early detection and intervention are crucial.

The gold standards of establishing the diagnosis of BED are the structured clinical interviews EDE (Cooper \& Fairburn, 1987) and SCID (First, Spitzer, Gibbon, \& Williams, 2002). In practice, diagnostic interviews require extensive training and are often lengthy and costly. Therefore selfreport scales are commonly used for screening purposes.

* Corresponding author at: University of Helsinki, Department of Public Health Biomedicum Helsinki 2B, 5th floor, 00014 University of Helsinki, Finland.

E-mail address: linda.mustelin@helsinki.fi (L. Mustelin).
There is no consensus regarding what is the best screening method for BED. The performance of a screening questionnaire against a diagnostic gold standard is often described by its sensitivity (true positive rate) and specificity (true negative rate). Several questionnaires have been tested as BED screens, but most of them either in samples contrasting healthy volunteers and clinically ill patients, or in highly selected samples of treatment-seeking individuals. The performance of diagnostic tests commonly varies across population subgroups; if studies testing the performance of diagnostic tests do not adequately represent all subgroups, spectrum bias can occur (Goehring, Perrier, \& Morabia, 2004). For this reason, it may be particularly important to assess how screening instruments perform in less selected, community-based samples, as the purpose of screening is to identify symptomatic individuals who could benefit from treatment but are not yet receiving it.

A widely available screening tool for eating disorders, the Eating Disorder Examination Questionnaire (EDE-Q), had a sensitivity of 73\% and specificity of $81 \%$ for screening BED in a small evaluation sample of patients with BED and control subjects without any binge eating (Vander Wal, Stein, \& Blashill, 2011). The same study evaluated the Binge Eating Disorder Test (BEDT) (sensitivity 100\%, specificity 100\%) and the Bulimia Test-Revised (BULIT-R) (sensitivity 100\%, specificity 96\%) (Vander Wal et al., 2011). The Binge Eating Scale (BES) had a sensitivity and specificity of $94 \%$ and $76 \%$ in bariatric surgery candidates (Grupski et al., 2013) and $85 \%$ and $20 \%$, respectively, in subjects with binge eating 
(Celio, Wilfley, Crow, Mitchell, \& Walsh, 2004). The Questionnaire for Eating and Weight Patterns-Revised (QEWP-R) had a sensitivity and specificity of $74 \%$ and $35 \%$ in subjects with binge eating (Celio et al., 2004 ), and $55 \%$ and $80 \%$, respectively, in women seeking treatment for obesity and/or binge eating (Borges, Morgan, Claudino, \& da Silveira, 2005). We identified only one study where screening was performed in a community sample: the Patient Health Questionnaire eating disorder module (PHQ-ED) yielded a sensitivity of $100 \%$ and a specificity of 92\% against diagnosis obtained by the Eating Disorder Examination in a community sample of young adult males and females (StriegelMoore et al., 2010).

The Eating Disorder Inventory (EDI) (Garner, 1991) has been frequently used as an eating disorder screening tool (Friborg, Clausen, \& Rosenvinge, 2013; Jacobi, Abascal, \& Taylor, 2004; Segura-Garcia et al., 2015) and measure of treatment response (Brambilla et al., 2009; Danielsen \& Ro, 2012; Fittig, Jacobi, Backmund, Gerlinghoff, \& Wittchen, 2008; Hagman et al., 2011; Lammers, Vroling, Ouwens, Engels, \& van Strien, 2015). It is available in many languages and discriminates well between eating disorder patients and both psychiatric and normal control subjects (Nevonen, Clinton, \& Norring, 2006). To our knowledge, however, no studies have assessed whether the EDI is an appropriate screening instrument for BED.

The aim of this study was to test whether EDI or its subscales can be used to screen for DSM-5 BED. To explore its screening properties, we compared the EDI and its subscales to clinician-conducted semistructured interviews (SCID) in a population setting.

\section{Methods}

\subsection{FinnTwin16 birth cohorts}

This nationwide cohort study of health behaviors in twins and their families (Kaprio, Pulkkinen, \& Rose, 2002) identified twin births in 1975-79 from the central population register of Finland. Data collection and analysis were approved by the ethics committee of the Department of Public Health of the University of Helsinki.

The twins and their parents were sent baseline self-report questionnaires when the twins were 16 y (wave 1). Follow-up questionnaires were mailed to the twins when they were 17 years (wave 2), 18 years (wave 3), 22-27 years (wave 4), and 31-37 years (wave 5). The analyses in this paper are based on wave 4 .

\subsection{Eating disorders diagnoses, wave 4}

At age 22-27 years, 2825 women (87\% of the original cohort) returned their questionnaire. The questionnaire included a self-report screen for eating disorder symptoms (Keski-Rahkonen et al., 2006; Mustelin et al., 2015). All screen-positive women $(\mathrm{N}=292)$, their screen-negative female co-twins $(\mathrm{N}=130)$, and a random sample of screen-negative women $(\mathrm{N}=210)$ were invited to participate in diagnostic telephone interviews. The overall interview participation rate was 86.7\% (Keski-Rahkonen et al., 2006; Mustelin et al., 2015).

Five experienced clinicians from the Eating Disorder Unit of Helsinki University Central Hospital conducted the interviews by telephone using the Structured Clinical Interview for DSM-IV (SCID-I) (First et al., 2002) to obtain current and lifetime diagnoses of anorexia nervosa, bulimia nervosa and BED. DSM-5 diagnoses for BED were established by recoding the DSM-IV SCID interviews as previously described (Mustelin et al., 2015). We identified 16 women suffering from BED as defined in DSM-5. Further details of the BED cases are presented elsewhere (Mustelin et al., 2015).

\subsection{Eating Disorder Inventory}

At wave 4 our participants completed four subscales of the EDI-2 (Garner, 1991): Body Dissatisfaction (Cronbach $\alpha=0.92$ ), Drive for
Thinness (Cronbach $\alpha=0.87$ ) Bulimia (Cronbach $\alpha=0.83$ ), and Perfectionism (Cronbach $\alpha=0.85$ ). The 6-point Likert scale was scored using the clinical scoring scheme $0-0-0-1-2-3$ as suggested by the manual. The global score of three EDI subscales was calculated as the sum of the Bulimia, Body Dissatisfaction, and Drive for Thinness subscales. The Perfectionism scores were available only from a subsample (393 individuals) because they were assessed in a separate questionnaire after the interviews.

\subsection{Assessment of screening properties}

The sensitivity of a test is the probability of a positive test result if one has the disorder. The specificity of a test is the probability of a negative test result if one does not have the disorder. The more sensitive a test, the more likely it is to identify all true cases, while a high specificity minimizes the number of false positives.

Sensitivity and specificity can only be calculated for a binary screen. Because our screening items were continuous, we plotted Receiver Operating Characteristic (ROC) curves. In ROC analysis, sensitivity is plotted against 1-specificity for each possible cutoff point of each screening item. The better the test distinguishes cases from noncases, the closer the curve follows the left hand and top borders of the graph. An instrument that performs no better than a guess has a ROC curve that follows the diagonal from the lower left to the upper right corner of the graph.

We formally compared the ROC curves of different screening measures by testing the differences of areas under the ROC curves with logistic linear predictors and the roccomp command in Stata 13. We used the Yoden method to determine the optimal cutoff-points (simultaneously maximizing both sensitivity and specificity) and calculated sensitivities and specificities for the dichotomized items.

\section{Results}

\subsection{Comparison of different EDI subscales}

Various approaches utilizing the EDI were appropriate for screening for DSM-5 BED. The approach that resulted in the best screening properties was the three-scale global score (AUC 0.86) (Fig. 1). However, three individual subscales were not statistically significantly worse in screening for BED (AUC 0.83, p = 0.54 for Bulimia; AUC 0.82, p = 0.08 for Body Dissatisfaction; AUC 0.81, $\mathrm{p}=0.09$ for Drive for Thinness, as compared to the three-scale global score). In contrast, the

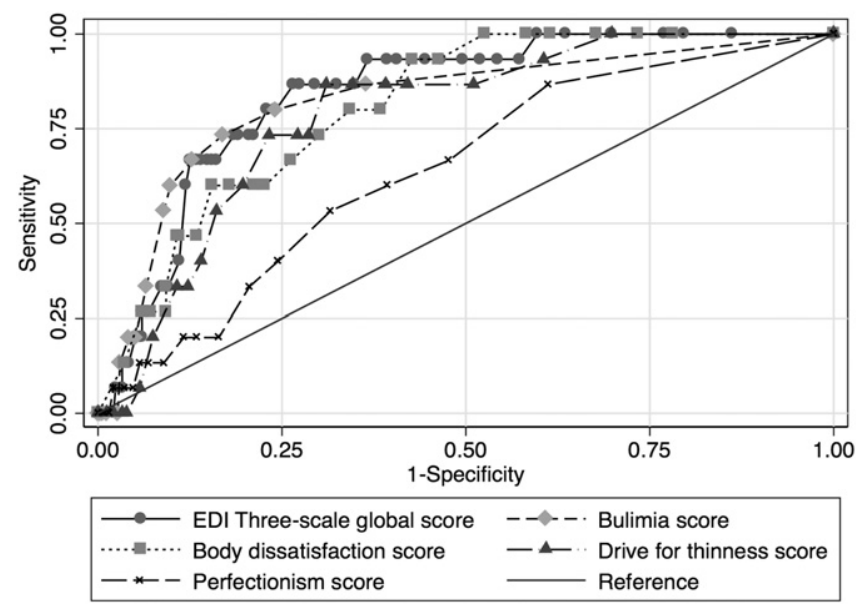

Fig. 1. ROC curves: EDI items predicting DSM-5 binge eating disorder. The best tradeoff between sensitivity and specificity was obtained using the three-scale global score (the sum of Bulimia, Drive for Thinness, and Body Dissatisfaction scores) or one of its subscales, whereas the Perfectionism scale performed significantly worse $(p=0.003)$. 
Perfectionism subscale was significantly worse as a screen for BED than the three-scale global score (AUC 0.64, $\mathrm{p}=0.003$ ).

\subsection{Optimal cutoff points}

To be clinically useful, a screening tool must have a cutoff point. One approach for determining cutoff points is to simultaneously maximize sensitivity and specificity. Using this approach, the sensitivity of the three-subscale global score was $87 \%$ and specificity $76 \%$ at cutoff $\geq 21$. The optimal cutoff points and corresponding sensitivities and specificities of the individual subscales are given in Table 1.

\section{Discussion}

Early detection of BED among non-treatment-seeking individuals is particularly important given that effective treatments are available and untreated BED has major adverse physical and mental health consequences. Our study suggests that the EDI-2 is suited for screening BED among young women. The three-scale global EDI score (the sum score of the Bulimia, Drive for Thinness and Body Dissatisfaction scales) was the best screen, but any of its three subscales could be used without apparent worsening in screening properties. The Perfectionism subscale had significantly worse screening properties. This concurs with research showing no association of perfectionist tendencies with clinical BED (Fairburn et al., 1998) or binge eating in the absence of compensatory behaviors (Forbush, Heatherton, \& Keel, 2007).

The choice of cutoff point for a continuous screen is always a tradeoff between sensitivity and specificity. The cutoff points presented in the paper were chosen to simultaneously maximize sensitivity and specificity. Using the ROC diagram (Fig. 1), other cutoff points can be chosen to minimize the chance of missing true cases (maximize sensitivity) or minimize false positives (maximize specificity). Importantly, positive screening results should never be interpreted as the presence of BED in the absence of a further assessment.

Several alternative instruments have been developed to screen for $\mathrm{BED}$, and many of them have reasonable screening properties (Borges et al., 2005; Celio et al., 2004; Grupski et al., 2013; Striegel-Moore et al., 2010; Vander Wal et al., 2011). However, we believe that the EDI has some important advantages. First, it is widely available and has been validated in many languages (Psychological Assessment Resources, 2015). Second, it performed well as a screening tool for BED as currently described in DSM-5. Third, it performed well in a sample where eating disorder presentations varied in severity and presentation. Initial validation samples are often 'black-and-white': participants are either healthy volunteers or patients from a specialist clinic. In reality, the process of screening is more nuanced: the distinction between disordered and healthy is not always straightforward. Our sample included women with BED, anorexia nervosa, bulimia nervosa, and subthreshold eating disorders. The sensitivities and specificities obtained in our study therefore reflect not only the ability of the EDI scales to distinguish women with BED from healthy women but also distinguishing them from women with other eating disorders.

Table 1

Screening properties of the EDI scales.

\begin{tabular}{lllll}
\hline EDI scale & $\begin{array}{c}\text { AUC } \\
\text { point }\end{array}$ & $\begin{array}{c}\text { Cut-off } \\
\text { ponsitivity }\end{array}$ & Specificity \\
\hline Global score of three subscales (Bulimia, & 0.86 & $\geq 21$ & $87 \%$ & $76 \%$ \\
$\quad$ Drive for Thinness, Body & & & & \\
$\quad$ Dissatisfaction) & 0.83 & $\geq 2$ & $80 \%$ & $78 \%$ \\
Bulimia & 0.81 & $\geq 8$ & $93 \%$ & $60 \%$ \\
Body Dissatisfaction & 0.82 & $\geq 7$ & $87 \%$ & $72 \%$ \\
Drive for Thinness & 0.64 & $\geq 1$ & $87 \%$ & $38 \%$ \\
Perfectionism & & & \\
\hline
\end{tabular}

EDI, Eating Disorder Inventory; AUC, area under the curve.
We are aware of just one study that tested a screening instrument for BED in a community setting. The PHQ-ED yielded a high sensitivity (100\%) and specificity (92\%) (Striegel-Moore et al., 2010) in a randomly selected community sample. The PHQ-ED is also shorter than the EDI, which is an advantage, as brevity increases the utility of a screening instrument both in community settings and the clinic. Another community-based study tested BES as a screen for clinically significant binge eating (Duarte, Pinto-Gouveia, \& Ferreira, 2015), yielding high sensitivity ( $82 \%$ ) and specificity (98\%). These instruments may be superior to the EDI and should be directly compared to the EDI in future studies. Recently, the EDI-3 has shown promising screening properties for anorexia nervosa and bulimia nervosa (Friborg et al., 2013) and for the presence of any eating disorder (Segura-Garcia et al., 2015) and should be evaluated for BED screening. More research is also needed on the potential benefits of screening for subthreshold BED, as well as instruments best suited for this purpose.

Our study was limited in several respects. Despite its large size, good participation and nationwide coverage, the number of BED diagnoses was small. Therefore statistical power for comparisons of different EDI subscales was limited. We also did not test the performance of the EDI subscales in an independent sample: future studies should attempt to do this. We used the EDI-2 rather than the most recent version (EDI3 ); future studies should confirm and expand our findings using the new version of EDI. Because our participants were young women from the community, they may differ considerably from men with BED as well as from individuals in special clinical populations, for example individuals assessed for bariatric surgery. Further, our sample was ethnically homogeneous, consisting of white Finnish women only; our results may not be generalizable to ethnically diverse populations, as eating disorder presentations may differ by race/ethnicity (Jennings, KellyWeeder, \& Wolfe, 2015). Finally, our participants were twins. Future studies should seek to confirm our findings in non-twin community samples.

Nevertheless, we believe that these limitations are offset by the strengths of our community-based setting, the diagnostic variety of the participants, and the use of the new DSM-5 diagnostic definition.

\section{Conclusions}

The EDI is widely available and routinely used for eating disorder assessment. It also performed well as a screening tool for BED in our community-based sample of young twin women. Future studies should assess its value in other populations and in clinical settings.

\section{Role of funding sources}

The data collection and analysis was supported by the Academy of Finland (grants 141054, 265240, 263278 and 264146 to JK and grant 259764 to AR). LM was supported by the Finnish Medical Foundation, the Yrjö Jahnsson Foundation, and the Medical Society of Finland (Finska Läkaresällskapet). The funding sources had no role in the study design, collection, analysis or interpretation of the data, writing the manuscript, or the decision to submit the paper for publication.

\section{Contributors}

L. Mustelin and A. Keski-Rahkonen designed the study, reviewed the literature, conducted the statistical analysis, and wrote the first draft of the manuscript. A. KeskiRahkonen supervised the interviewers and conducted part of the clinical interviews. U. Kärkkäinen helped to come up with the idea of the study and contributed to the analyses. J. Kaprio supervised the twin cohort data collection. All authors contributed to and have approved the final manuscript.

\section{Conflict of interest}

The authors declare that they have no conflicts of interest.

\section{Acknowledgements}

The data collection and analysis was supported by the Academy of Finland (grants 141054, 265240, 263278 and 264146 to JK and grant 259764 to AR). LM was supported by the Finnish Medical Foundation, the Yrjö Jahnsson Foundation, and the Medical Society of Finland (Finska Läkaresällskapet). 


\section{References}

American Psychiatric Association (2013). The diagnostic and statistical manual of mental disorders: DSM-5 (5th ed.). Washington D.C.: American Psychiatric Association.

Berkman, N. D., Brownley, K. A., Peat, C. M., Lohr, K. N., Cullen, K. E., Morgan, L. C., et al. (2015). doi:NBK338312 [book accession].

Borges, M. B., Morgan, C. M., Claudino, A. M., \& da Silveira, D. X. (2005). Validation of the portuguese version of the questionnaire on eating and weight patterns-revised (QEWP-R) for the screening of binge eating disorder. Revista Brasileira De Psiquiatria (Sao Paulo, Brazil: 1999), 27(4), 319-322 (doi:S1516-44462005000400012 [pii]).

Brambilla, F., Samek, L., Company, M., Lovo, F., Cioni, L., \& Mellado, C. (2009). Multivariate therapeutic approach to binge-eating disorder: Combined nutritional, psychological and pharmacological treatment. International Clinical Psychopharmacology, 24(6), 312-317. http://dx.doi.org/10.1097/YIC.0b013e32832ac828 (doi).

Bulik, C. M., Sullivan, P. F., \& Kendler, K. S. (2002). Medical and psychiatric morbidity in obese women with and without binge eating. The International Journal of Eating Disorders, 32(1), 72-78 10.1002/eat.10072 [doi]).

Celio, A. A., Wilfley, D. E., Crow, S. J., Mitchell, J., \& Walsh, B. T. (2004). A comparison of the binge eating scale, questionnaire for eating and weight patterns-revised, and eating disorder examination questionnaire with instructions with the eating disorder examination in the assessment of binge eating disorder and its symptoms. The International Journal of Eating Disorders, 36(4), 434-444 (10.1002/eat.20057 [doi]).

Cooper, Z., \& Fairburn, C. (1987). The eating disorder examination: A semi-structured interview for the assessment of the specific psychopathology of eating disorders. International Journal of Eating Disorders, 6(1), 1-8. http://dx.doi.org/10.1002/1098108X(198701)6:1<1::AID-EAT2260060102>3.0.CO;2-9.

Danielsen, M., \& Ro, O. (2012). Changes in body image during inpatient treatment for eating disorders predict outcome. Eating Disorders, 20(4), 261-275. http://dx.doi.org/10. 1080/10640266.2012.689205 (doi).

Duarte, C., Pinto-Gouveia, J., \& Ferreira, C. (2015). Expanding binge eating assessment: Validity and screening value of the binge eating scale in women from the general population. Eating Behaviors, 18, 41-47 (doi:S1471-0153(15)00039-2 [pii])

Fairburn, C. G., Doll, H. A., Welch, S. L., Hay, P. J., Davies, B. A., \& O'Connor, M. E. (1998). Risk factors for binge eating disorder: A community-based, case-control study. Archives of General Psychiatry, 55(5), 425-432.

First, M. B., Spitzer, R. L., Gibbon, M., \& Williams, J. B. (2002). Structured clinical interview for DSM-IV-TR axis I disorders, research version, non-patient edition (SCID-I/NP). New York: Biometrics Research, New York State Psychiatric Institute.

Fittig, E., Jacobi, C., Backmund, H., Gerlinghoff, M., \& Wittchen, H. U. (2008). Effectiveness of day hospital treatment for anorexia nervosa and bulimia nervosa. European Eating Disorders Review: The Journal of the Eating Disorders Association, 16(5), 341-351 (10. 1002/erv.883 [doi]).

Forbush, K., Heatherton, T. F., \& Keel, P. K. (2007). Relationships between perfectionism and specific disordered eating behaviors. The International Journal of Eating Disorders, 40(1), 37-41 (10.1002/eat.20310 [doi]).

Friborg, O., Clausen, L., \& Rosenvinge, J. H. (2013). A five-item screening version of the eating disorder inventory (EDI-3). Comprehensive Psychiatry, 54(8), 1222-1228 (10. 1016/j.comppsych.2013.05.004 [doi]).

Garner, D. M. (1991). Eating disorder inventory-2, professional manual. Odessa, FL: Psychological Assessment Resources.

Goehring, C., Perrier, A., \& Morabia, A. (2004). Spectrum bias: A quantitative and graphical analysis of the variability of medical diagnostic test performance. Statistics in Medicine, 23(1), 125-135 (10.1002/sim.1591 [doi]).

Grupski, A. E., Hood, M. M., Hall, B. J., Azarbad, L., Fitzpatrick, S. L., \& Corsica, J. A. (2013). Examining the binge eating scale in screening for binge eating disorder in bariatric surgery candidates. Obesity Surgery, 23(1), 1-6. http://dx.doi.org/10.1007/s11695011-0537-4 (doi).

Hagman, J., Gralla, J., Sigel, E., Ellert, S., Dodge, M., Gardner, R., et al. (2011). A doubleblind, placebo-controlled study of risperidone for the treatment of adolescents and young adults with anorexia nervosa: A pilot study. Journal of the American Academy of Child and Adolescent Psychiatry, 50(9), 915-924. http://dx.doi.org/10.1016/j.jaac. 2011.06.009 (doi)

Hudson, J. I., Lalonde, J. K., Coit, C. E., Tsuang, M. T., McElroy, S. L., Crow, S. J., et al. (2010). Longitudinal study of the diagnosis of components of the metabolic syndrome in individuals with binge-eating disorder. The American Journal of Clinical Nutrition, 91(6), 1568-1573. http://dx.doi.org/10.3945/ajcn.2010.29203 (doi).

Jacobi, C., Abascal, L., \& Taylor, C. B. (2004). Screening for eating disorders and high-risk behavior: Caution. The International Journal of Eating Disorders, 36(3), 280-295 (10. 1002/eat.20048 [doi]).

Jennings, K. M., Kelly-Weeder, S., \& Wolfe, B. E. (2015). Binge eating among racial minority groups in the united states: An integrative review. Journal of the American Psychiatric Nurses Association, 21(2), 117-125. http://dx.doi.org/10.1177/ 1078390315581923 (doi).

Kaprio, J., Pulkkinen, L., \& Rose, R. J. (2002). Genetic and environmental factors in healthrelated behaviors: Studies on Finnish twins and twin families. Twin Research: The Official Journal of the International Society for Twin Studies, 5(5), 366-371. http://dx. doi.org/10.1375/136905202320906101.

Keski-Rahkonen, A., Sihvola, E., Raevuori, A., Kaukoranta, J., Bulik, C. M., Hoek, H. W., et al (2006). Reliability of self-reported eating disorders: Optimizing population screening. The International Journal of Eating Disorders, 39(8), 754-762. http://dx.doi.org/10 1002/eat.20277.

Lammers, M. W., Vroling, M. S., Ouwens, M. A., Engels, R. C., \& van Strien, T. (2015). Predictors of outcome for cognitive behaviour therapy in binge eating disorder. European Eating Disorders Review: The Journal of the Eating Disorders Association, 23(3), 219-228 (10.1002/erv.2356 [doi]).

Mond, J. M., Hay, P. J., Rodgers, B., \& Owen, C. (2007). Health service utilization for eating disorders: Findings from a community-based study. The International Journal of Eating Disorders, 40(5), 399-408 (10.1002/eat.20382 [doi]).

Mussell, M. P., Mitchell, J. E., Weller, C. L., Raymond, N. C., Crow, S. J., \& Crosby, R. D (1995). Onset of binge eating, dieting, obesity, and mood disorders among subjects seeking treatment for binge eating disorder. The International Journal of Eating Disorders, 17(4), 395-401.

Mustelin, L., Raevuori, A., Hoek, H. W., Kaprio, J., \& Keski-Rahkonen, A. (2015). Incidence and weight trajectories of binge eating disorder among young women in the community. The International Journal of Eating Disorders, 48(8), 1106-1112 (10.1002/eat. 22409 [doi]).

Nevonen, L., Clinton, D., \& Norring, C. (2006). Validating the EDI-2 in three Swedish female samples: Eating disorders patients, psychiatric outpatients and normal controls. Nordic Journal of Psychiatry, 60(1), 44-50 (doi:KJ06R11H515N7504 [pii]).

Psychological Assessment Resources (2015). Eating disorder inventory. (Retrieved 5/25 from http://www4.parinc.com/products/PermsLicensing.aspx?id=12).

Segura-Garcia, C., Aloi, M., Rania, M., Ciambrone, P., Palmieri, A., Pugliese, V., et al. (2015) Ability of EDI-2 and EDI-3 to correctly identify patients and subjects at risk for eating disorders. Eating Behaviors, 19, 20-23 (doi:S1471-0153(15)00079-3 [pii]).

Striegel-Moore, R. H., Cachelin, F. M., Dohm, F. A., Pike, K. M., Wilfley, D. E., \& Fairburn, C. G. (2001). Comparison of binge eating disorder and bulimia nervosa in a community sample. The International Journal of Eating Disorders, 29(2), 157-165. http://dx.doi. org/10.1002/1098-108X(200103)29:2<157::AID-EAT1005>3.0.CO;2-8 (pii).

Striegel-Moore, R. H., Perrin, N., DeBar, L., Wilson, G. T., Rosselli, F., \& Kraemer, H. C. (2010). Screening for binge eating disorders using the patient health questionnaire in a community sample. The International Journal of Eating Disorders, 43(4), 337-343 (10.1002/eat.20694 [doi]).

Vander Wal, J. S., Stein, R. I., \& Blashill, A. J. (2011). The EDE-Q, BULIT-R, and BEDT as selfreport measures of binge eating disorder. Eating Behaviors, 12(4), 267-271. http://dx. doi.org/10.1016/j.eatbeh.2011.07.006 (doi). 